\title{
Growth, biofilm formation, antifungal susceptibility and oxidative stress resistance of Candida glabrata are affected by different glucose concentrations
}

\begin{abstract}
Glucose is an important fuel source to support many living organisms. Its importance in the physiological fitness and pathogenicity of Candida glabrata, an emerging human fungal pathogen has not been extensively studied. The present study aimed to investigate the effects of glucose on the growth, biofilm formation, antifungal susceptibility and oxidative stress resistance of C. glabrata. In addition, its effect on the expression of a putative high affinity glucose sensor gene, SNF3 was also investigated. Glucose concentrations were found to exert effects on the physiological responses of C. glabrata. The growth rate of the species correlated positively to the amount of glucose. In addition, low glucose environments were found to induce C. glabrata to form biofilm and resist amphotericin B. Conversely, high glucose environments promoted oxidative stress resistance of C. glabrata. The expression of CgSNF3 was found to be significantly up-regulated in low glucose environments. The expression of SNF3 gene in clinical isolates was found to be higher compared to ATCC laboratory strains in low glucose concentrations, which may explain the better survivability of clinical isolates in the low glucose environment. These observations demonstrated the impact of glucose in directing the physiology and virulence fitness of C. glabrata through the possible modulation by SNF3 as a glucose sensor, which in turn aids the species to adapt, survive and thrive in hostile host environment.
\end{abstract}

Keyword: Candida glabrata; Glucose; Growth; Biofilm formation; Antifungal susceptibility; Oxidative stress resistance 